\title{
Mid-infrared Variability of Changing-look AGNs
}

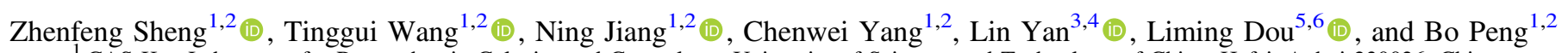 \\ ${ }^{1}$ CAS Key Laboratory for Researches in Galaxies and Cosmology, University of Sciences and Technology of China, Hefei, Anhui 230026, China \\ shengzf@mail.ustc.edu.cn \\ ${ }^{2}$ School of Astronomy and Space Science, University of Science and Technology of China, Hefei 230026, China; twang@ustc.edu.cn \\ ${ }^{3}$ Caltech Optical Observatories, Cahill Center for Astronomy and Astrophysics, California Institute of Technology, Pasadena, CA 91125, USA \\ ${ }^{4}$ Infrared Processing and Analysis Center, California Institute of Technology, Pasadena, CA 91125, USA \\ ${ }^{5}$ Center for Astrophysics, Guangzhou University, Guangzhou 510006, China \\ ${ }^{6}$ Astronomy Science and Technology Research Laboratory of Department of Education of Guangdong Province, Guangzhou 510006, China \\ Received 2017 May 23; revised 2017 July 10; accepted 2017 August 10; published 2017 August 29
}

\begin{abstract}
It is known that some active galactic nuclei (AGNs) transit from Type 1 to Type 2 or vice versa. There are two explanations for the so-called changing-look AGNs: one is the dramatic change of the obscuration along the line of sight, and the other is the variation of accretion rate. In this Letter, we report the detection of large amplitude variations in the mid-infrared luminosity during the transitions in 10 changing-look AGNs using the Wide-field Infrared Survey Explorer (WISE) and newly released Near-Earth Object WISE Reactivation data. The mid-infrared light curves of 10 objects echo the variability in the optical band with a time lag expected for dust reprocessing. The large variability amplitude is inconsistent with the scenario of varying obscuration, rather it supports the scheme of dramatic change in the accretion rate.
\end{abstract}

Key words: accretion, accretion disks - galaxies: active - galaxies: Seyfert - infrared: galaxies

\section{Introduction}

Active galactic nuclei (AGNs) are empirically classified into Type 1 and Type 2 according to the emission line widths. Type 1 AGNs show both broad and narrow emission lines in spectra while Type 2 display only narrow lines. Intermediate types, 1.5 and 1.8/1.9, were further introduced depending on the relative strength of broad and narrow lines (Osterbrock 1977, 1981). Whereas the early discovery of broad lines in the polarized spectra of Type 2 AGNs (Antonucci \& Miller 1985), together with other evidence, led to a unification scheme (Antonucci 1993): two types of AGNs are intrinsically the same but differ only in the orientation of the torus-like obscurer. In this scheme, Type 1 AGNs are viewed face-on so that we look directly into the central accretion disk and the broad emission line region (BLR), while Type 2 AGNs are viewed edge-on and our line of sight to the central engine is blocked by a putative dusty torus. Despite the success of the unification model, there are arguments that at least some Type 2 AGNs are intrinsic, lacking broad lines because of an inadequate accretion rate (Shi et al. 2010; Bianchi et al. 2012; Pons \& Watson 2014).

Some AGNs are known to transit between Type 1 and Type 2 (e.g., from Type 2 to Type 1, Khachikian \& Weedman 1971; from Type 1 to Type 2, Penston \& Perez 1984). These objects are called changing-look (CL) AGNs, featuring emerging or disappearing broad emission lines (BELs). There are some notable CL AGNs reported so far. Mrk 590 changed from Seyfert 1.5 to 1.0 and back to 2 over several decades (Denney et al. 2014). NGC 2617 was reported to have changed from Type 1.8 to Type 1 (Shappee et al. 2014), but recently it likely has a new outburst and continues brightening (Oknyansky et al. 2017). More recently, it has been reported that Mrk 1018 changed back to Type 1.9 after 30 years of being Type 1 (McElroy et al. 2016).

Although the origin of the CL behavior is not well understood, various scenarios have been proposed. In one scenario, CL is interpreted in the context of the unification scheme, and disappearing or emerging of BELs are ascribed to the variable obscurer moving in and out of the line of sight (Goodrich 1989). In the other scenario, CL is attributed to the dramatic changes in accretion rate, arising from disk instability or even the tidal disruption, in which the continuum and broad lines should respond immediately while narrow lines remain nearly unchanged. LaMassa et al. (2015) demonstrated that both the photometric and spectral properties of CL AGN J0159 +0033 cannot be explained by the unification paradigm, but suggested that accretion power decreases. MacLeod et al. (2016) undertook a systematic search for CL AGNs using SDSS and Pan-STARRS1 and found 10 of them. Runnoe et al. (2016) recently reported a new CL AGN, J1011+5442, through the Time Domain Spectroscopic Survey. Both MacLeod et al. (2016) and Runnoe et al. (2016) favor the interpretation of accretion rate change.

In this work, we focus on the mid-infrared variability (MIR) of CL AGNs and its application in testing CL scenarios. Because the infrared emission is produced by dust heated by the UV radiation of accretion disk, it would respond to the variation of the latter with a time lag of order of years. Jun et al. (2015) used the midinfrared echo to confirm that PG 1302-102's optical periodic variability is accretion disk driven. Besides, infrared emission is much less affected by dust extinction than optical radiation as the opacity decreases steeply toward long wavelength. Moreover, the size of the torus is much larger than those of the BLR and accretion disk. Therefore, the effect of obscuration by a dusty cloud in the optical and infrared would be very different. This would allow us to test the two different scenarios.

We report a discovery of a significant infrared variation of eight CL AGNs. The outline of this Letter is as follows. In Section 2, we describe the Catalina Real-Time Transient Survey (CRTS) data, Wide-field Infrared Survey Explorer (WISE)/Near-Earth Object WISE Reactivation (NEOWISE) data, and Sloan Digital Sky Survey (SDSS) data used in this study, along with initial data processing. In Section 3, we present some details of each source and shortly review their properties. In Section 4, we simply discuss the possible 
Table 1

Information of 24 Changing-look AGNs

\begin{tabular}{|c|c|c|c|c|c|c|c|c|}
\hline Name & $\begin{array}{c}t_{\mathrm{spec}} \\
(\mathrm{MJD}) \\
(2)\end{array}$ & $\begin{array}{c}\Delta t_{\text {wise }- \text { spec }} \\
\quad \text { (year) } \\
\text { (3) }\end{array}$ & $\begin{array}{c}\operatorname{Max} \Delta W 1 \\
(\operatorname{mag}) \\
(4)\end{array}$ & $\begin{array}{c}\operatorname{Max} \Delta W 2 \\
(\operatorname{mag}) \\
(5)\end{array}$ & (6) & $\begin{array}{c}\sigma_{W 2} \\
\text { (7) }\end{array}$ & $\begin{array}{c}\text { Transition } \\
\text { (8) }\end{array}$ & $\begin{array}{l}\text { Note } \\
(9)\end{array}$ \\
\hline J002311.06+003517.5 & 51816,55480 & -0.29 & $0.47 \pm 0.03$ & $0.28 \pm 0.04$ & 13.62 & 6.89 & A BELs & MacLeod et al. (2016) \\
\hline J015957.64+003310.4 & 51871,55201 & 0.03 & $0.21 \pm 0.06$ & $0.26 \pm 0.12$ & 3.67 & 2.28 & D BELs & LaMassa et al. (2015) \\
\hline J012648.08-083948.0 & 52163,54465 & 2.53 & $0.06 \pm 0.04$ & $0.16 \pm 0.08$ & 1.72 & 1.87 & D BELs & Ruan et al. (2016) \\
\hline J022556.07+003026.7 & 52944,55445 & -0.11 & $0.23 \pm 0.04$ & $0.39 \pm 0.12$ & 5.31 & 3.30 & D \& A BELs & MacLeod et al. (2016) \\
\hline J022652.24-003916.5 & 52641,56267 & -2.88 & $0.23 \pm 0.06$ & $0.48 \pm 0.14$ & 3.53 & 3.53 & D BELs & MacLeod et al. (2016) \\
\hline J035301.02-062326.3 & 51908,54853 & 1.05 & $0.18 \pm 0.02$ & $0.24 \pm 0.03$ & 10.36 & 9.14 & $1.8 \rightarrow 1$ & Runco et al. (2016) \\
\hline J081319.34+460849.5 & 51877,55210 & 0.24 & $0.33 \pm 0.01$ & $0.57 \pm 0.02$ & 27.47 & 31.42 & $1.8 \rightarrow 1$ & Runco et al. (2016) \\
\hline J084748.28+182439.9 & 53711,54852 & 1.26 & $0.29 \pm 0.01$ & $0.34 \pm 0.02$ & 22.19 & 14.93 & $1 \rightarrow 1.9 \rightarrow 2$ & Runco et al. (2016) \\
\hline J090902.35+133019.4 & 53826,55210 & 0.29 & $0.70 \pm 0.02$ & $1.20 \pm 0.05$ & 41.98 & 24.57 & $1.8 \rightarrow 1$ & Runco et al. (2016) \\
\hline J093812.27+074340.0 & 52733,55210 & 0.32 & $0.04 \pm 0.01$ & $0.07 \pm 0.01$ & 4.26 & 4.76 & $1 \rightarrow 1.8$ & Runco et al. (2016) \\
\hline J094838.43+403043.5 & 52709,55211 & 0.29 & $0.19 \pm 0.01$ & $0.16 \pm 0.01$ & 20.94 & 15.41 & $1 \rightarrow 1.8$ & Runco et al. (2016) \\
\hline $\mathrm{J} 100220.17+450927.3$ & 52376,56683 & -3.74 & $0.30 \pm 0.03$ & $0.26 \pm 0.06$ & 9.36 & 4.58 & D BELs & MacLeod et al. (2016) \\
\hline J101152.98+544206.4 & 52652,57073 & -4.82 & $1.17 \pm 0.04$ & $1.76 \pm 0.08$ & 33.19 & 22.69 & D BELs & Runnoe et al. (2016) \\
\hline $\mathrm{J} 102152.34+464515.6$ & 52614,56769 & -3.97 & $0.65 \pm 0.03$ & $0.73 \pm 0.03$ & 25.68 & 21.27 & D BELs & MacLeod et al. (2016) \\
\hline $\mathrm{J} 132457.29+480241.2$ & 52759,56805 & -3.97 & $0.45 \pm 0.02$ & $0.47 \pm 0.02$ & 27.55 & 17.65 & D BELs & MacLeod et al. (2016) \\
\hline $\mathrm{J} 154507.53+170951.1$ & 53889,54936 & 0.82 & $0.53 \pm 0.01$ & $0.66 \pm 0.01$ & 49.72 & 47.89 & $1.8 \rightarrow 1$ & Runco et al. (2016) \\
\hline $\mathrm{J} 155440.25+362952.0$ & 53172,57543 & -6.34 & $0.67 \pm 0.02$ & $0.96 \pm 0.03$ & 35.93 & 27.76 & $2 \rightarrow 1$ & Gezari et al. (2017) \\
\hline J214613.31+000930.8 & 52968,55478 & -0.39 & $0.14 \pm 0.04$ & $0.13 \pm 0.06$ & 3.48 & 2.20 & A BELs & MacLeod et al. (2016) \\
\hline $\mathrm{J} 225240.37+010958.7$ & 52174,55500 & -0.40 & $0.65 \pm 0.06$ & $0.88 \pm 0.09$ & 11.26 & 9.93 & A BELs & MacLeod et al. (2016) \\
\hline J233317.38-002303.4 & 52199,55447 & -0.23 & $0.28 \pm 0.04$ & $0.14 \pm 0.05$ & 7.45 & 2.81 & A BELs & MacLeod et al. (2016) \\
\hline J233602.98+001728.7 & 52096,55449 & -0.23 & $0.40 \pm 0.08$ & $0.71 \pm 0.25$ & 5.20 & 2.78 & D BELs & Ruan et al. (2016) \\
\hline Mrk 590 & 52649,56664 & -3.97 & $0.13 \pm 0.01$ & $0.34 \pm 0.01$ & 18.61 & 43.95 & $1.5 \rightarrow 1 \rightarrow 2$ & Denney et al. (2014) \\
\hline Mrk 1018 & 51812,57033 & -4.99 & $0.76 \pm 0.01$ & $1.05 \pm 0.01$ & 79.92 & 105.78 & $1.9 \rightarrow 1 \rightarrow 1.9$ & McElroy et al. (2016) \\
\hline NGC 2617 & 53003,56407 & -2.99 & $0.64 \pm 0.01$ & $0.87 \pm 0.01$ & 74.38 & 93.95 & $1.8 \rightarrow 1$ & Shappee et al. (2014) \\
\hline
\end{tabular}

Note. The information of 24 CL AGNs. $t_{\text {spec }}$ lists the MJD of two spectra be used to confirm the type transition; $\Delta t_{\text {wise-spec }}$ lists the interval between first $W I S E$ data point and the spectrum epoch (second MJD in $\left.t_{\text {spec }}\right)$ that confirmed transition $\left(\triangle t_{\text {wise-spec }}<0\right.$ means that the transition is more likely to to be covered by WISE/ NEOWISE); columns 4 and 5 list the maximum variation of the $W 1$ and $W 2$ bands, while columns 6 and 7 are the corresponding variation significance of $W 1$ and $W 2$; column 8 lists the transition of each source, A BELs means appear, while D BELs means disappear; the last column lists the corresponding reference.

scenarios, and then we come to a conclusion in Section 5. We adopt a flat $\Lambda$ CDM cosmology with $H_{0}=70 \mathrm{~km} \mathrm{~s}^{-1} \mathrm{Mpc}^{-1}$ and $\Omega_{m}=0.27$.

\section{Data}

In this section, we will introduce the data sets used to construct the optical/MIR light curves and the sample selection. Our investigation is mainly based on $V$-band data from CRTS (Drake et al. 2009) and MIR data from WISE (Wright et al. 2010) and the newly released Near-Earth Object WISE Reactivation mission (NEOWISE-R; Mainzer et al. 2014). CRTS is one of the largest time domain optical surveys currently operating, covering $\sim 33,000 \mathrm{deg}^{2}$ with a baseline of 8 years and $\sim 250$ exposures per year for each target. The survey is performed using unfiltered light but calibrated to a $V$-band zeropoint. We rejected the data points with large uncertainties ( $>0.2 \mathrm{mag}$ ) and spurious points (usual outliers). Then, we binned these data using the median value. The WISE has surveyed the full sky 1.2 times in four infrared bands, $W 1$, $W 2, W 3$, and $W 4$, centered at 3.4, 4.6, 12, and $22 \mu \mathrm{m}$ from 2010 January to September, on which its cryogen used to cool the W3 and W4 instruments was exhausted. Afterward it was extended an additional four months using $W 1$ and $W 2$, and then it was placed in hibernation on 2011 February 1. On 2013 October 3, it was reactivated and named NEOWISE-R, using only $W 1$ and $W 2$ (Mainzer et al. 2014). So there is a $\sim 3.5$ year gap in both the W1 and W2 band light curves. WISE scans a fullsky area every half year and thus yielded 6-7 times the observations for each object up to the most recent public catalog. First, we removed bad data points of low image quality ("qi_fact" $<1$ ) and with a small separation to the South Atlantic Anomaly ("SAA" $<5$ ), and we flagged moon masking ("moon_mask" = 1). Then, we grouped the data by every half year as in our previous work (Jiang et al. 2012, 2016) and binned the data using the median value. Besides that we also collected the Stripe 82 multi-epoch data (Abazajian et al. 2009) to visually compare to the CRTS $V$-band data.

We collect the CL AGNs reported in the literature as much as possible to investigate their MIR behavior. These sources are then screened according to following criteria:

(1) Seyfert galaxies or quasars that changed from Type 1 to Type $\geqslant 1.8$ or from Type $\geqslant 1.8$ to Type 1 . Immediate types, 1.2 or 1.5 , are abandoned because they may be partially obscured, complicating the interpretation.

(2) Variability amplitude is larger than $0.4 \mathrm{mag}$ at $10 \sigma$ significance in either the W1or/and W2 bands.

(3) No source contamination within 6". The angular resolution is 6". 1 and 6" 4 at $W 1$ and W2 (Wright et al. 2010). We check the SDSS image for each object to exclude source contamination.

Twenty-four CL AGNs that satisfied criterion (1) are listed in Table 1, among which, 11 objects meet the requirement (2). Finally, 10 follow all of these criteria, which are named SDSS J002311.06+003517.5, SDSS J081319.34+460849.5, SDSS J090902.35+133019.4, SDSS J101152.98+544206.4, SDSS J102152.34+464515.6, SDSS J132457.29+480241.2, SDSS J155440.25+362952.0 (iPTF 16bco), SDSS J225240.37 
(a)

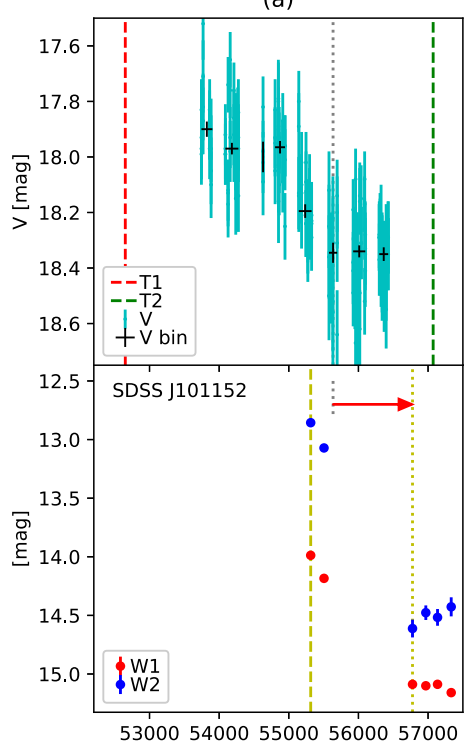

(e)

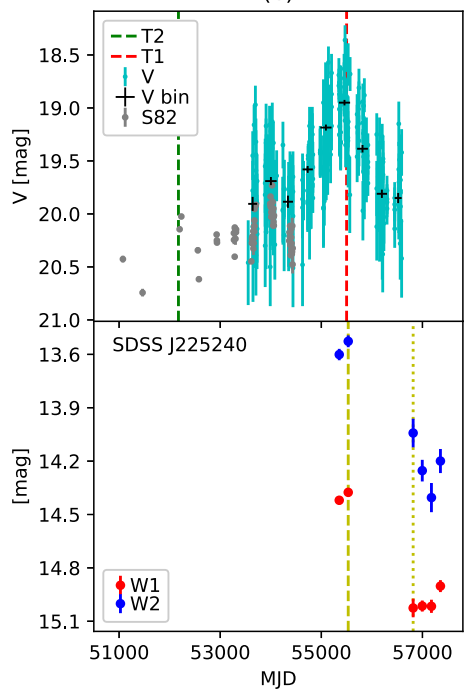

(b)

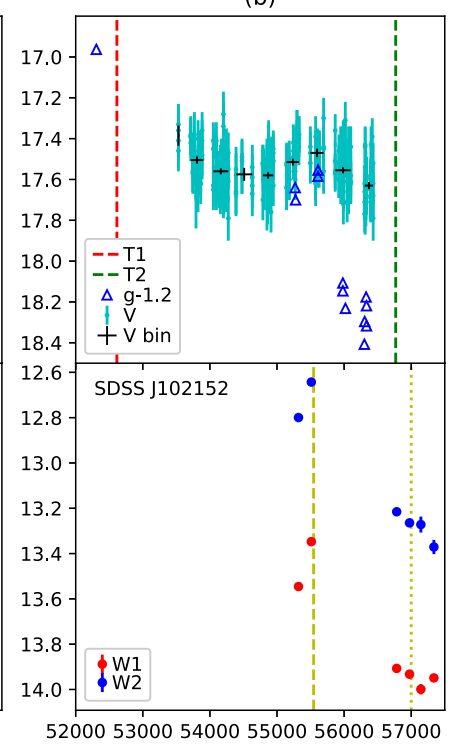

(f)

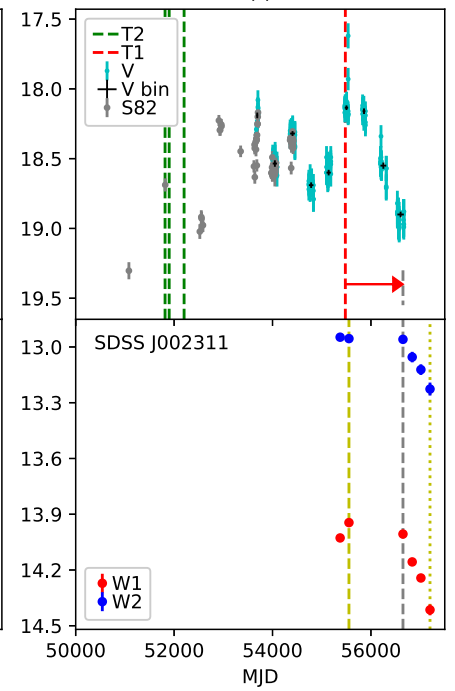

(c)

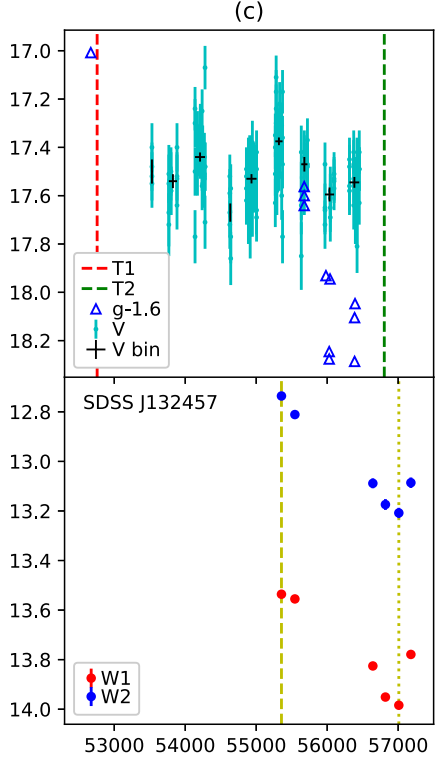

(g)

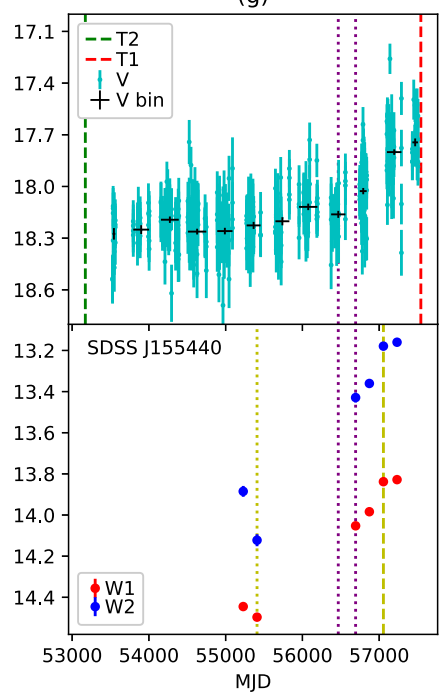

(d)

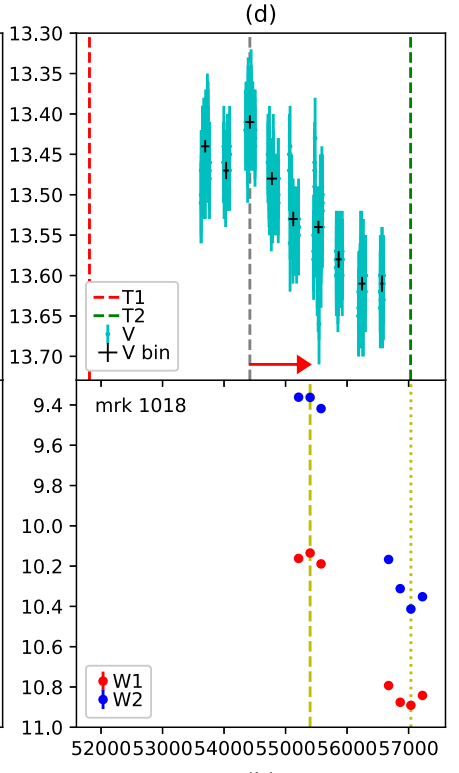

(h)

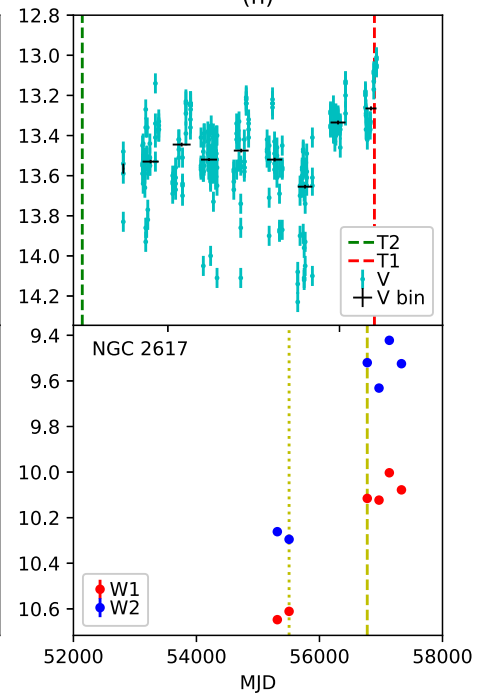

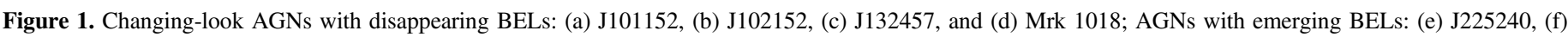

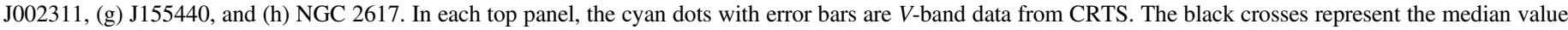

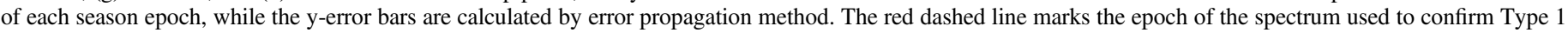

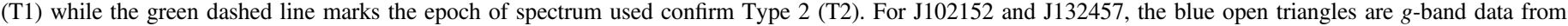

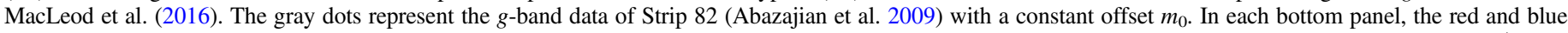

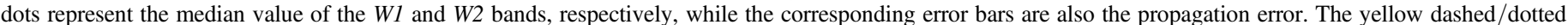

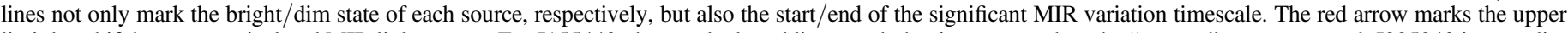

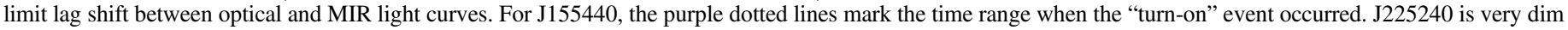
in the $V$-band, so we constrain the CRTS measurement error $\leqslant 0.4$ when performing data processing.

+010958.7 (hereafter J002311, J081319, J090902, J101152, $\mathrm{J} 102152$, J132457, J155440, and J225240, respectively), Mrk 1018, and NGC 2617.

\section{Mid-Infrared and Optical Light Curves}

The 10 sources with significant MIR variability can be categorized into two equal subsamples depending on whether the BELs appeared or disappeared, that is changed from Type 2 to Type 1 or vice versa. Below, we will introduce the MIR and optical light curves of the two classes, respectively.

\subsection{From Type 1 to Type 2}

$\mathrm{J} 101152$, J102152, J132457, and Mrk 1018 are reported to experience transitions from Type 1 to Type $>1.8$ (MacLeod et al. 2016; McElroy et al. 2016; Runnoe et al. 2016). Their CRTS and WISE light curves are presented in Figures 1(a)-(d). Along with the transition, all the MIR light curves show apparent dimming ( $>0.4 \mathrm{mag}$ ) in both $W 1$ and $W 2$. However, only J101152 and Mrk 1018 exhibit a similar dimming trend in the $V$-band light curves. We have tried to fit their SDSS images with the PSF+Sersic model using 2D decomposition software GALFIT (Peng et al. 2002), assuming that the PSF and Sersić represent the AGN and host galaxy emission, respectively. Our results suggest that the PSF component accounts for $26 \%$ and $42 \%$ of $\mathrm{J} 102152$ and $\mathrm{J} 132457$ in the SDSS $r$-band, which is taken before the type transition. Due to seeing limit, the fitted PSF component can be considered as an upper limit of the AGN, which means that the real AGN fraction is even lower, and thus their optical variability is largely diluted. We have 

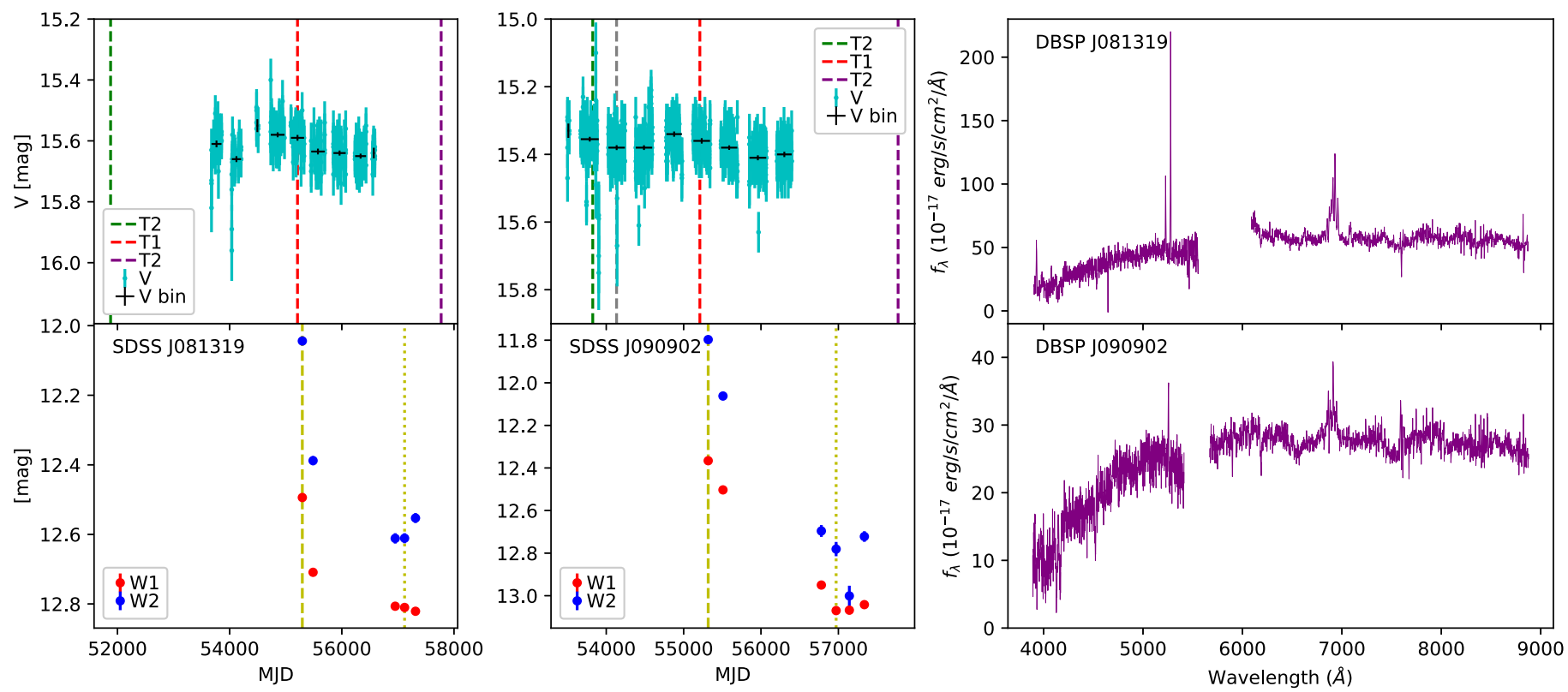

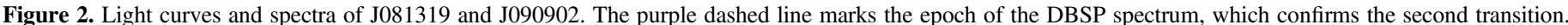

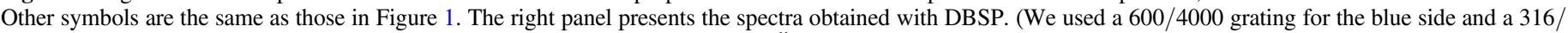

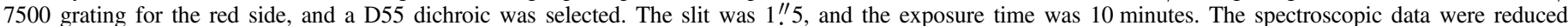
following the IRAF standard routine.)

also noted that their $g$-band variability is much more significant ( $\Delta g>1 \mathrm{mag}$; MacLeod et al. 2016) because it is less affected by the host galaxy. J101152 is totally AGN dominated and Mrk 1018 is a nearby $(z=0.035)$ Seyfert galaxy with a wellresolved nucleus, making their $V$-band variation pretty detectable. We use yellow dashed and dotted lines to mark the significant/upper limit variation timescale of MIR bands of each source (see Figure 1).

Here, we provide some notes for each object.

(1) J101152: Wl faded by $1.10 \mathrm{mag}$ within $\sim 4.00$ years, while $W 2$ faded by 1.76 mag.

(2) J102152: in $\sim 4.01$ years, $W 1$ and $W 2$ dimmed $\sim 0.59$ and $\sim 0.62$ mag respectively.

(3) J132457: it showed the disappearance of BELs (MacLeod et al. 2016), but recently showed the likely appearances of BELs (Ruan et al. 2016). Two MIR bands show similar re-brightened behavior. Both MIR bands dimmed continuously with $W 1$ and $W 2 \sim 0.47$ and $\sim 0.45 \mathrm{mag}$, respectively, in $\sim 4.53$ years and then brightened after the turning point $\mathrm{MJD} \simeq 57000$.

(4) Mrk 1018: it changed from Type 1.9 to Type 1 in 1984 (Cohen et al. 1986), but recently returned to Type 1.9 (MacLeod et al. 2016). In 4.49 years, Wl dimmed by 0.76 mag while $W 2$ dimmed by 1.05 mag.

\subsection{From Type 2 to Type 1}

In Figures 1(e)-(h), we plot J225240, J002311, J155440, and NGC 2617, which show evidence of emerging BELs (Shappee et al. 2014; MacLeod et al. 2016; Gezari et al. 2017). Along with the transition, J155440 and NGC 2617 show a rising trend in their MIR bands. As for J225240 and J002311, the BOSS spectrum was taken during the WISE epoch, which means their transitions happened before the WISE survey (see panels (e) and (f)). So the WISE and NEOWISE missed the main uptrend transition period but covered the latter period. This situation is more obvious in J081319 and J090902, which are presented in Figure 2. We note the six objects as follows.

(1) J225240. $V$-band luminosity kept increasing $(\Delta V>$ $1 \mathrm{mag}$ ) during the transition, but decreased afterward, indicating it might change back to a dim state. W1 and $W 2$ exhibit very similar variations. Because there is a gap between WISE and NEOWISE, we can only derive an upper limit of decreasing timescale as 3.52 years, with $W 1$ and $W 2$ dimming $\sim 0.65$ and $\sim 0.52$ mag, respectively.

(2) J002311. Optical/MIR variation behavior is very similar to that of J2252. The MIR bands dropped significantly in 1.51 years (from the gray dashed line to the yellow dotted line), with $W 1$ and $W 2$ dimming $\sim 0.41$ and $\sim 0.27 \mathrm{mag}$, respectively). Due to the gap in the MIR bands, we estimate the upper limit of decreasing timescale to be 4.51 years (from the yellow dashed line to the yellow dotted line).

(3) J155440. This was discovered as a transient on 2016 June 1 by iPTF (named "iPTF 16bco"; Gezari et al. 2017). From MJD $=55409$ to 57055 , during $\sim 4.51$ years $W 1$ and $W 2$ brightened 0.69 and $0.94 \mathrm{mag}$, respectively.

(4) NGC 2617. This was a Seyfert 1.8 galaxy in 2003 but showed the appearance of BELs in 2013 (Shappee et al. 2014). From MJD $=55506$ to 56776 , during $\sim 3.48$ years the $W 1$ and W2 brightened 0.5 and $0.78 \mathrm{mag}$, respectively.

(5) J081319 and J090902.J081319 was a Seyfert 1.8 galaxy in 2000, so was J090902 in 2006. Both of them were classified as Type 1 in 2010 (Runco et al. 2016). Their MIR bands present a remarkable drop, contrary to their previous transition, indicating a new change. We confirmed they have changed back to Type 1.9 using the Double Spectrograph (DBSP) of the Hale 200 inch telescope at Palomar Observatory. The spectrum of J081319 was obtained on 2017 January 8, while that of J090902 was obtained on January 18 (see Figure 3).

In summary, MIR bands of all the objects show a very similar variation to the optical data, which is in accordance with the type transition. In Figure 3, we plot $W 1-W 2$ of each source. The sources with a transition from Type 1 to Type 2 likely 


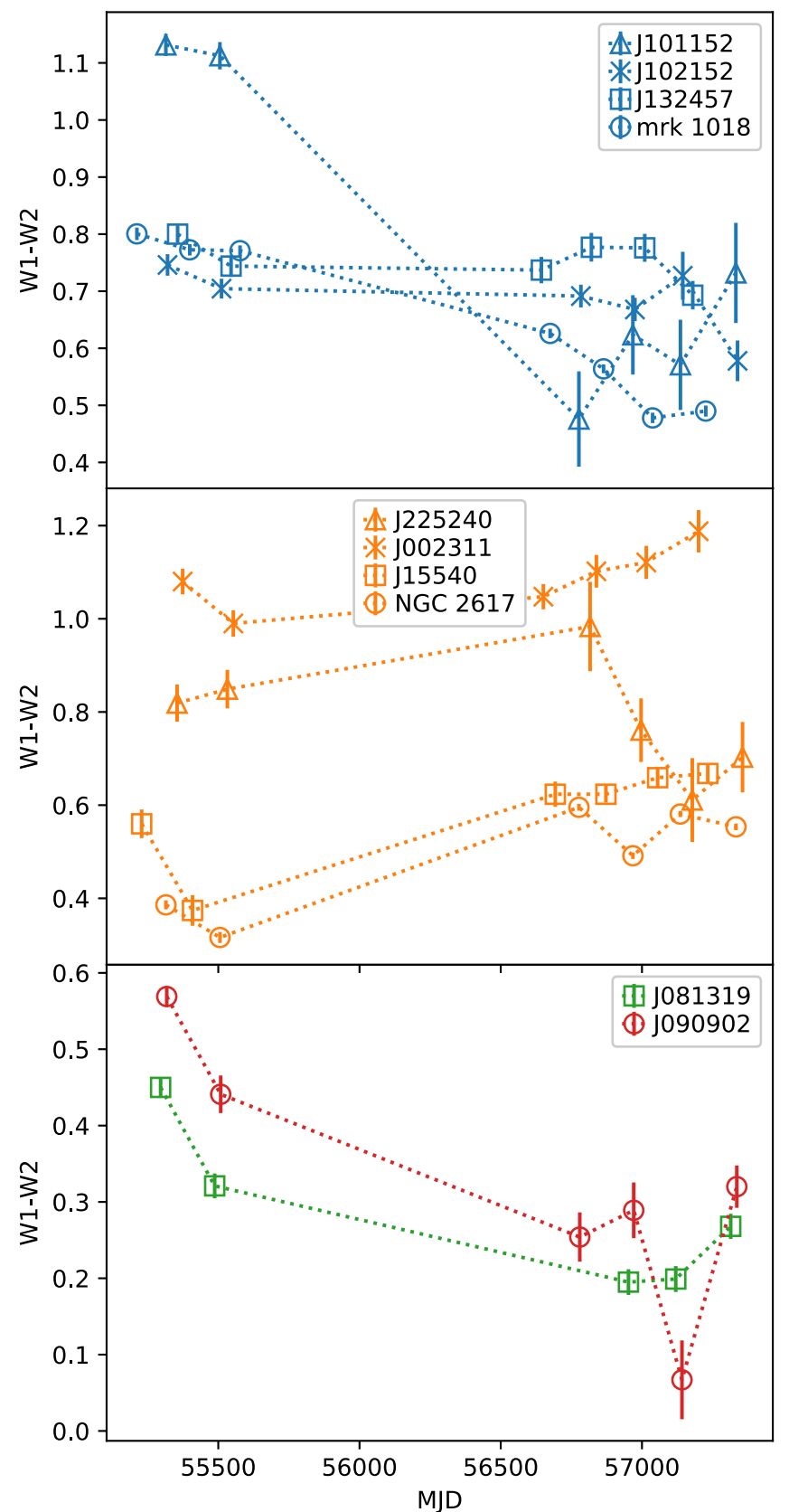

Figure 3. Color change of changing-look AGNs. Upper panel: changing from Type 1 to Type 2-like; middle panel: changing from Type 2-like to Type 1; bottom panel: J0813 and J0909.

change from AGN-like MIR-color $(W 1-W 2>0.8)$ to galaxylike MIR-color $(W 1-W 2<0.5)$, and vice versa (Stern et al. 2012; Yan et al. 2013). We list the upper limits of MIR variation time as $\Delta T$ in column 9 and the change of MIR bands $\Delta W 1$ and $\Delta W 2$ in columns 11 and 12 of Table 2.

\subsection{Remaining Objects}

Besides the 10 sources with significant MIR variability described above, we also examine the remaining targets with very weak/non-detectable MIR variations. For example, $\mathrm{J} 233317.38-002303.4$ and $\mathrm{J} 214613.31+000930.8$, which have the appearance of BELs, show a significant uptrend in $g$-band light curves but turn into a plateau with little variation after MJD 54500 (MacLeod et al. 2016), implying they might finish the transition. For Mrk 590, it changed to Type $1.8 \sim 1.9$ by 2006 (Denney et al. 2014). Since their MIR light curves began at MJD 55179, WISE likely missed their most significant transition epoch. For J233602.98+001728.7 and J022652.24-003916.5 their infrared radiations are too faint $(W 1 \sim 15.4, W 2 \sim 14.8)$ to detect significant variation. The reasons above are applicable to most of the other objects not included in our sample, except J154507.53+170951.1, which is contaminated by another source within $6 "$.

\section{Discussion \\ 4.1. Physical Scenarios}

The main motivation of this Letter is to explore the physical mechanism of CL AGNs using MIR variability. MIR emission at 3.4 and $4.6 \mu \mathrm{m}$ are mainly originated from hot-dust emission heated by AGNs (Netzer 2013); hence, the MIR emission should respond to the variation of accretion rate with a time delay. On the other hand, the MIR bands are not significantly affected by dust extinction; any detectable variability means a much larger amplitude of variability in the optical if CL is caused by the changing of obscuration. Even for J002311, whose MIR variation is the weakest $(\Delta W 2=0.27)$ among the 10 objects in our sample, $\Delta V \sim 6$ is required when assuming the extinction model of Fitzpatrick \& Massa (1999). Such a dramatic optical variability is extremely rare for AGNs and does not agree with the optical light curves here.

Nevertheless, we further investigate the dynamical timescale of the obscuration. The size of the obscurer should be at least comparable to the torus to block the hot dust. With the inner radius of the torus simply estimated from the dust sublimation radius (Netzer 2013; $R_{\text {sub }}=0.5 L_{46}^{0.5}\left(1800 \mathrm{~K} / T_{\text {sub }}\right) \mathrm{pc}$ ), the crossing time for the obscuring material can be derived as $t_{\text {cross }}=0.073\left[r_{\text {orb }} /(\text { lt-day })\right]^{3 / 2} M_{8}^{-0.5} \arcsin \left(r / r_{\text {orb }}\right)$ year (LaMassa et al. 2015), where $r_{\text {orb }}$ is the circular orbital radius of the obscurer, $M_{8}$ is the mass of black hole in units of $10^{8} M_{\odot}$, and $r$ is the true size of the obscured region (i.e., the continuum emitting region or BLR size). We adopted $r$ as the BLR size, which is estimated from the calibrated $R-L$ relation (Bentz et al. 2013), and the calculated $R_{\text {sub }}$ and $t_{\text {cross }}$ are listed in Table 2 (columns 6 and 8). It can be seen clearly that $t_{\text {cross }}$ is much longer than the observed MIR variation time $\Delta T$ for all 10 objects.

Based on the analysis above, the CL behavior of our sample cannot be a result of the changes in obscuration. Once the obscuration case is ruled out, the MIR variability can be naturally attributed the hot-dust echo of the dramatic changing of the accretion rate. The time delay of the MIR and optical variability can offer us a unique opportunity to measure the radius of the torus. Previous studies basing on $K$-band reverberation mapping suggested $R_{\mathrm{in}}=R_{\tau_{K}}=0.47\left(L_{\mathrm{bol}} / 10^{46} \mathrm{erg} \mathrm{s}^{-1}\right)^{0.5}$ (Suganuma et al. 2006). Assuming $R_{\lambda}=\left(\lambda / \lambda_{K}\right)^{2} R_{\tau_{K}}$, we got $R_{W 1}=0.36 \mathrm{pc} \quad(0.11 \mathrm{pc})$ and $R_{W 2}=0.67 \mathrm{pc} \quad(0.21 \mathrm{pc})$ for $\log L_{\mathrm{bol}}=45 \mathrm{erg} \mathrm{s}^{-1}\left(44 \mathrm{erg} \mathrm{s}^{-1}\right)$, corresponding to a time lag of 2.18 years ( 0.68 years) in the rest frame, or $1 \sim 3$ years in the observed frame. We have tried to shift the MIR light curves backward a few of years, their variation pattern matching the optical ones well, giving fantastic evidence for the dust echo response to the accretion. In summary, we conclude that all $10 \mathrm{CL}$ AGNs with significant MIR variability have undergone an drastic drop/rise in accretion rate. 
Table 2

Properties of Changing-look AGNs

\begin{tabular}{|c|c|c|c|c|c|c|c|c|c|c|c|}
\hline Name & $\begin{array}{l}z \\
\text { (2) }\end{array}$ & $\begin{array}{c}\log M_{\mathrm{BH}} / M_{\odot} \\
\text { (3) }\end{array}$ & $\begin{array}{c}\log L_{\mathrm{bol}} \\
\left(\mathrm{erg} \mathrm{s}^{-1}\right) \\
(4)\end{array}$ & $\begin{array}{c}\log L_{5100} \\
\left(\mathrm{erg} \mathrm{s}^{-1}\right) \\
(5)\end{array}$ & $\begin{array}{l}R_{\text {sub }} \\
(\mathrm{pc}) \\
(6)\end{array}$ & $\begin{array}{c}R_{\mathrm{BLR}} \\
\text { (1t-day) } \\
(7)\end{array}$ & $\begin{array}{c}t_{\text {cross }} \\
\text { (year) } \\
(8)\end{array}$ & $\begin{array}{c}\Delta T \\
\text { (year) } \\
(9)\end{array}$ & $\begin{array}{l}R_{\text {torus }} \\
(\mathrm{pc}) \\
(10)\end{array}$ & $\begin{array}{c}\Delta W 1 \\
(\mathrm{mag}) \\
(11)\end{array}$ & $\begin{array}{c}\Delta W 2 \\
(\mathrm{mag}) \\
(12)\end{array}$ \\
\hline J002311.06+003517.5 & 0.422 & 9.23 & 45.480 & 44.513 & 0.275 & 63.16 & 28.95 & 4.51 & 0.69 & 0.41 & 0.27 \\
\hline J101152.98+544206.4 & 0.246 & 7.78 & 45.117 & 44.150 & 0.181 & 40.45 & 69.96 & 4.00 & $\ldots$ & 1.10 & 1.76 \\
\hline J102152.34+464515.6 & 0.204 & 8.33 & 45.121 & 44.154 & 0.182 & 40.65 & 36.16 & 4.01 & 0.81 & 0.59 & 0.62 \\
\hline $\mathrm{J} 132457.29+480241.2$ & 0.272 & 8.51 & 45.303 & 44.336 & 0.224 & 50.83 & 43.11 & 4.53 & $\ldots$ & 0.45 & 0.47 \\
\hline $\mathrm{J} 155440.25+362952.0$ & 0.237 & $8^{*}$ & $45.146^{*}$ & $44.23^{*}$ & 0.187 & 44.63 & 60.61 & 4.51 & $\cdots$ & 0.66 & 0.94 \\
\hline J225240.37+010958.7 & 0.534 & 8.88 & 45.318 & 44.352 & 0.228 & 51.83 & 34.93 & 3.52 & $\ldots$ & 0.65 & 0.52 \\
\hline Mrk 1018 & 0.035 & $7.4 \sim 7.9^{*}$ & $44.491^{*}$ & & 0.088 & $24^{*}$ & $21.0 \sim 37.4$ & 4.49 & 0.82 & 0.76 & 1.05 \\
\hline NGC 2617 & 0.00142 & $7.6^{*}$ & $44.03^{*}$ & $43.12^{*}$ & 0.05 & 11.42 & 10.4 & 3.48 & $\ldots$ & 0.50 & 0.78 \\
\hline J081319.34+460849.5 & 0.054 & $6.98 \sim 7.28^{\star}$ & $43.56 \sim 44.01^{\star}$ & $42.65 \sim 43.10^{\star}$ & $0.03 \sim 0.05$ & $6.44 \sim 11.14$ & $9.57 \sim 15.34$ & 5.00 & $\ldots$ & 0.32 & 0.57 \\
\hline J090902.35+133019.4 & 0.050 & $7.03 \sim 7.32^{\star}$ & $43.42 \sim 43.87^{\star}$ & $42.51 \sim 42.96^{\star}$ & $0.03 \sim 0.04$ & $5.43 \sim 9.39$ & $7.06 \sim 11.31$ & 4.53 & $\ldots$ & 0.70 & 0.98 \\
\hline
\end{tabular}

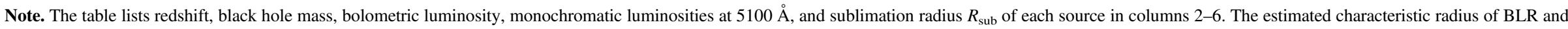

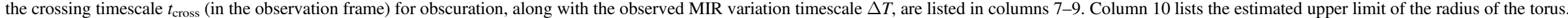

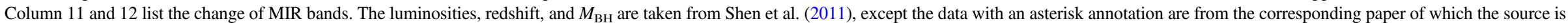
reported, and the data with a star annotation are estimated from $\mathrm{H} \alpha$ (Greene \& Ho 2005) by fitting the SDSS spectrum. 


\subsection{More Information from the Infrared Variation}

The MIR light curves can not only help us diagnose the physical scenarios of CL AGNs, but can also allow us to get more transition information and even predict new $\mathrm{CL}$ behaviors. Because MIR bands are AGN-heated hot-dust dominated, they are more efficient to detect when the AGN is optically weak comparable to its host galaxy (e.g., J102152 and J132457 in Section 3.1). For J155440, it was proposed that the turn-on change state occurred less than 500 days before 2016 June 1 (Gezari et al. 2017). However, according to the $V$ band and the MIR-band light curves, the turn-on event much likely began around 2013 June $\sim 2014$ February (marked by purple dotted lines; see Figure 1(g)). The two objects J081319 and J090902 presented a very similar sign of the new transition due to the MIR decline, and we confirmed they changed back to Type 1.9. For J225240 and J002311, which changed from Type 2-like to Type 1, show a dramatic decline in MIR and optical light curves, suggesting they might also have changed back to Type 2. We are planning to perform follow-up spectral observations to confirm our conjecture.

\section{Conclusion}

We investigate the 10 reported CL AGNs confirmed in the optical spectrum in the literature. Combining with WISE and NEOWISE multi-epoch photometric data $(W 1, W 2)$, all 10 sources have obvious MIR variation $(>0.4 \mathrm{mag})$ at a level $>10 \sigma$. The obscurer passing across the line of sight could not cause such a large variation, due to the extinction and dynamical obscuration timescale that failed to support it. Four sources with disappearance of BELs, namely, J101152, J102152, J132457, and Mrk 1018, show a similar significant decline $(>10 \sigma)$ in MIR light curves that echo the optical variation. We suggest that their $\mathrm{CL}$ is owing to a drop in the accretion rate. Among the four sources showing emerging BELs, two objects, J155440 and NGC 2617, feature a remarkable increase in MIR luminosity, in accordance with their transition and also the increasing $V$-band tendency. We suggest their CL is due to accretion rate rising up. Two other objects, J225240 and J002311, abnormally display a significant decrease in the MIR bands, indicating they might undergo a second transition and change back to the previous Type 2, which should be confirmed by follow-up observations. In particular, J081319 and J090902, which are reported to have changed from Type 1.8 to Type 1 , have a significant $(>10 \sigma)$ decrease in MIR signals. We confirmed that they changed back to Type 2. Further and repeated spectroscopic monitoring of sources with large MIR variability sources could be worthwhile.

We acknowledge the anonymous referee for valuable comments that helped to improve the Letter. And we acknowledge Andrew Drake for providing the unreleased CRTS data (Drake et al. 2009) of J155440. Also, we acknowledge Jordan N. Runco for helpful information on J081319 (Runco et al. 2016). We thank Luming Sun for discussion and Zhihao Zhong for his perfect spectrum reduction skill. This project is supported by National Basic Research Program of China (grant No. 2015CB857005), the NSFC through NSFC-11233002, NSFC-11421303, NSFC-116203021, and U1431229, jointly supported by the Chinese Academy of Science and NSF. This research has made use of the NASA/IPAC Infrared Science Archive, which is operated by the Jet Propulsion Laboratory, California Institute of Technology, under contract with the National Aeronautics and Space Administration. The CSS survey is funded by the National Aeronautics and Space Administration under grant No. NNG05GF22G issued through the Science Mission Directorate Near-Earth Objects Observations Program. The CRTS survey is supported by the U.S. National Science Foundation under grant No. AST-0909182. This research made use of Astropy, a community-developed core Python package for Astronomy (Astropy Collaboration et al. 2013).

\section{ORCID iDs}

Zhenfeng Sheng (1) https://orcid.org/0000-0001-6938-8670

Tinggui Wang (i) https://orcid.org/0000-0002-1517-6792

Ning Jiang (1) https://orcid.org/0000-0002-7152-3621

Lin Yan (1) https://orcid.org/0000-0003-1710-9339

Liming Dou (1) https://orcid.org/0000-0002-4757-8622

\section{References}

Abazajian, K. N., Adelman-McCarthy, J. K., Agüeros, M. A., et al. 2009, ApJS, 182, 543

Antonucci, R. 1993, ARA\&A, 31, 473

Antonucci, R. R. J., \& Miller, J. S. 1985, ApJ, 297, 621

Astropy Collaboration, Robitaille, T. P., Tollerud, E. J., et al. 2013, A\&A, 558, A33

Bentz, M. C., Denney, K. D., Grier, C. J., et al. 2013, ApJ, 767, 149

Bianchi, S., Panessa, F., Barcons, X., et al. 2012, MNRAS, 426, 3225

Cohen, R. D., Puetter, R. C., Rudy, R. J., Ake, T. B., \& Foltz, C. B. 1986, ApJ, 311,135

Denney, K. D., De Rosa, G., Croxall, K., et al. 2014, ApJ, 796, 134

Drake, A. J., Djorgovski, S. G., Mahabal, A., et al. 2009, ApJ, 696, 870

Fitzpatrick, E. L., \& Massa, D. 1999, ApJ, 525, 1011

Gezari, S., Hung, T., Cenko, S. B., et al. 2017, ApJ, 835, 144

Goodrich, R. W. 1989, ApJ, 340, 190

Greene, J. E., \& Ho, L. C. 2005, ApJ, 630, 122

Jiang, N., Dou, L., Wang, T., et al. 2016, ApJL, 828, L14

Jiang, N., Zhou, H.-Y., Ho, L. C., et al. 2012, ApJL, 759, L31

Jun, H. D., Stern, D., Graham, M. J., et al. 2015, ApJL, 814, L12

Khachikian, E. Y., \& Weedman, D. W. 1971, ApJL, 164, L109

LaMassa, S. M., Cales, S., Moran, E. C., et al. 2015, ApJ, 800, 144

MacLeod, C. L., Ross, N. P., Lawrence, A., et al. 2016, MNRAS, 457, 389

Mainzer, A., Bauer, J., Cutri, R. M., et al. 2014, ApJ, 792, 30

McElroy, R. E., Husemann, B., Croom, S. M., et al. 2016, A\&A, 593, L8

Netzer, H. 2013, The Physics and Evolution of Active Galactic Nuclei (Cambridge: Cambridge Univ. Press)

Oknyansky, V. L., Gaskell, C. M., Huseynov, N. A., et al. 2017, MNRAS, 467, 1496

Osterbrock, D. E. 1977, ApJ, 215, 733

Osterbrock, D. E. 1981, ApJ, 249, 462

Peng, C. Y., Ho, L. C., Impey, C. D., \& Rix, H.-W. 2002, AJ, 124, 266

Penston, M. V., \& Perez, E. 1984, MNRAS, 211, 33P

Pons, E., \& Watson, M. G. 2014, A\&A, 568, A108

Ruan, J. J., Anderson, S. F., Cales, S. L., et al. 2016, ApJ, 826, 188

Runco, J. N., Cosens, M., Bennert, V. N., et al. 2016, ApJ, 821, 33

Runnoe, J. C., Cales, S., Ruan, J. J., et al. 2016, MNRAS, 455, 1691

Shappee, B. J., Prieto, J. L., Grupe, D., et al. 2014, ApJ, 788, 48

Shen, Y., Richards, G. T., Strauss, M. A., et al. 2011, ApJS, 194, 45

Shi, Y., Rieke, G. H., Smith, P., et al. 2010, ApJ, 714, 115

Stern, D., Assef, R. J., Benford, D. J., et al. 2012, ApJ, 753, 30

Suganuma, M., Yoshii, Y., Kobayashi, Y., et al. 2006, ApJ, 639, 46

Wright, E. L., Eisenhardt, P. R. M., Mainzer, A. K., et al. 2010, AJ, 140, 1868

Yan, L., Donoso, E., Tsai, C.-W., et al. 2013, AJ, 145, 55 